\title{
STUDY of the SURFACE PROPERTIES Of CERAMIC MATERIALS \\ DY CHROMATOGRAPHY
}

Grant DE-FG05-88ER13859

Principal Investigator

Georges Guiochon

Department of Chemistry,

University of Tennessee, Knoxville, TN.

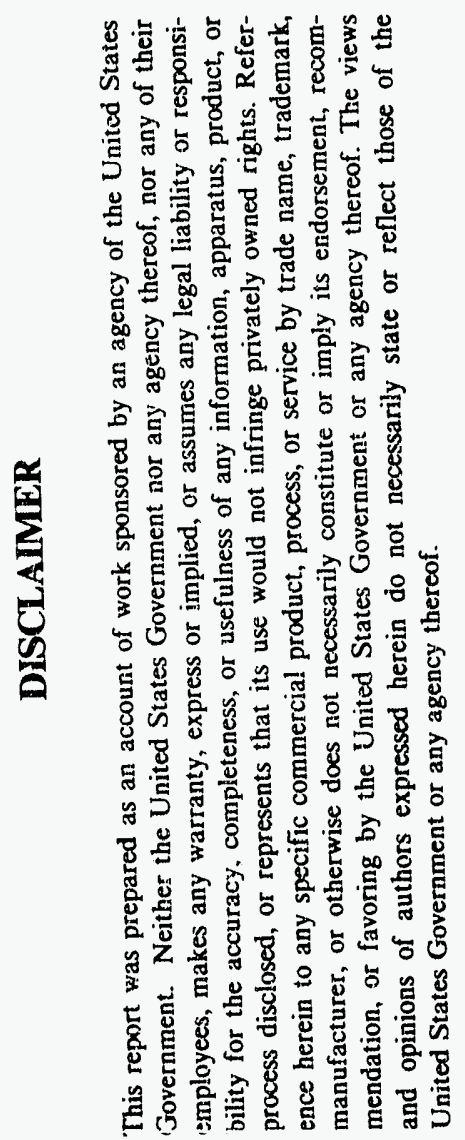

FINAL PERFORMANCE REPORT

March 1992 


\section{STUDY of the SURFACE PROPERTIES Of CERAMIC MATERIALS}

bY CHROMATOGRAPHY

FINAL PERFORMANCE REPORT, March 1992

Table of contents

ABSTRACT . . . . . . . . . . . . . . . . . . . . . . 3

INTRODUCTION •. . . . . . . . . . . . . . . . . . . . 4

I - Scientific objectives of the original Proposal . • . . . . 4

A. Aim of the Research. . . . . . . . . . . . . . . 5

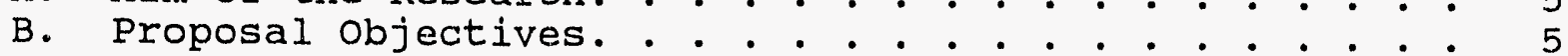

C. Evaluation of Technical Requirements. . . . . . . . 6

II - REPORT OF TECHNICAL PROGRESS . . . . . . . . . . . . . . . 7

A. Organization of the Research. . . . . . . . . . . . . 8

B. Instrument Modifications. . . . . . . . . . . . . 8

C. Column Preparation. . . . . . . . . . . . . . . 8

1. Description of the Preparation Procedure. . . . . 8

2. Reproducibility of the Experimental Results. . . . 9

D. Experimental Design and Data Estimation. . . . . . . . 10

1. Detector Calibration. . . . . . . . . . . . 10

2. Quantitation of the Desorption Recovery. . . . . . 10

E. 3. Determination of the Adsorption Isotherms. . . • . 11

Dorption Isotherm and the Energy Distribution

Function. . . . . . . . . . . . . . . . . . 12

1. Theoretical Model of Adsorption Energy Distribu-

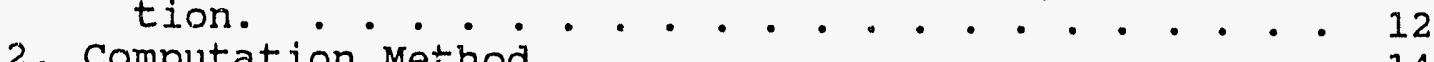

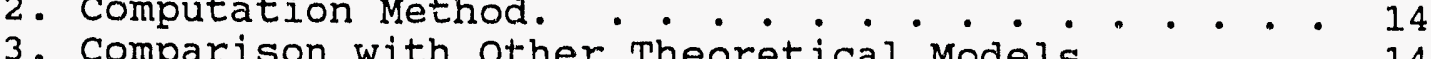

F. Examples of Application. . . . $\cdot \cdot \cdot 14$

G. Summary of Project Status. . . . . . . . . . . . . . . 15

LIST of PUBLICATIONS SUPPORTED bY GRANT DE-FG05-88ER13859 • • . 17 


\author{
STUDY of the SURFACE 'PROPERTIES Of CERAMIC MATERIALS \\ bY CHROMATOGRAPHY
}

FINAL PERFORMANCE REPORT, March 1992

\begin{abstract}
This report summarizes the progress made on the subject project during the grant period $(3 / 1 / 88$ to $2 / 29 / 92)$. During this period, we have:

(i) developed a manufacturing procedure of capillary columns with an inner wall coated with the ceramic particles whose surface is under study;

(ii) demonstrated the reproducibility of this procedure;

(iii) developed a method for the rapid determination of adsorption isotherms from the profiles of large sample bands;

(iv) developed the theoretical model permitting the calculation of the adsorption energy distribution from the isotherm, while making fewer assumptions than current methods and no arbitrary ones;

(v) coded, tested and validated the calculation procedure developed, which involved solving serious, unexpected computational difficulties;

(vi) prepared a series of columns made with alumina particles of various origins:

(vii) determined the adsorption energy distributions of diethylether and 1-chlorobutane on the surfaces of these powders;

(viii) demonstrated a correlation between the process quality of ceramic alumina and the adsorption energy distribution of 1-chlorobutane;

(ix) begun the collection of data on silica particles; and

(x) begun a comparison between the various procedures published in the literature to derive the adsorption energy distribution from the experimental isotherm.
\end{abstract}




\section{Study of the Surface Properties of Ceramic Materials by Chromatography}

\section{Final Performance Report, March 1992}

\section{INTRODUCTION}

In this report, we first review the aim of the original proposal and the research plan for the four years starting in March 1988, in order to provide a perspective for the work carried out. Then, we describe the theoretical and experimental work performed, the results achieved, and the present status of the project.

In summary, we consider that we have essentially achieved our original objectives and made a significant contribution to the understanding of the fundamentals of adsorption on real surfaces. Most fundamental research on adsorption assumes adsorbent surfaces to be homogeneous. Material chemists have been able to design and prepare a variety of adsorbents with a highly homogeneous surface. These adsorbents are widely used in chromatography and in adsorption studies, contributing to further anchor the idea that homogeneous surfaces are the most frequent case. In reality, many common materials used as finely divided powders such as pigments, fillers, ceramics, or involved ir industrial procedures such as adhesion, have inhomogeneous surfaces, and this fact explains difficulties and troubles in predicting their properties and usefulness. The main result of our work is a practical method which permits the detailed study of the chemical properties of non-homogeneous surfaces.

\section{I - Scientific objectives of the original proposal}

our research grant supported investigations of the surface properties of ceramic powders by inverse gas chromatography. Specialty ceramic products have achieved a great importance, notably in the production of high temperature materials for aeronautic engines and of materials with unusual dielectric, magnetic or electric: properties for electronic devices.

Considerable difficulties are met when molding and firing ceramic parts. The raw material is a fine powder (particle size between 0.2 and $1 \mu \mathrm{m}$ ) of the desired oxide, nitride or carbide. After mixing with other powders (flux), a binding agent (usually polymeric), a solvent and a dispersant (surface active agent), the powder is agglomerated and molded, then fired. During the thermal process, the parts or green bodies (originally porous, unconsolidated agglomerates) shrink, and their porosities decrease from about $40 \%$ to almost zero. The resulting changes in volume may generate internal stress, resulting in the rupture of the part or the formation of cracks. During the firing, the organic compounds introduced to facilitate the molding of the green body and to give it the strength 
to withhold handling stress are vaporized and/or pyrolyzed. If vaporization is too slow, extensive pyrolysis may result in the deposition of traces of carbon and the loss of mechanical strength of the parts, or the loss of other performance (e.g., electrical). Ceramic raw powders having the same origin and the same known properties behave sometimes quite differently, some giving satisfactory products, others not.

Growing evidence has accumulated that this phenomenon is due to differences in the surface chemistry of the particles which are not shown by conventional tests, such as titration curves, the zeta potential, the $\mathrm{pH}$ of a suspension of the powder, etc. Because major changes in the chemical properties of atoms or group of atoms on the surface involve relatively minor changes in their bonding energy to the solid, physical methods using irradiation with high energy particles to abstract these atoms from the surface and analyze them are not well suited to compare lots of similar particles of different origins. Thus, other methods of studying the chemical properties of the surface appear to be necessary.

\section{A. Aim of the Research.}

The aim of our research was the development of a general method of characterization of surface heterogeneity based on the determination of the adsorption isotherms of carefully selected probe components on the surface of the studied material, for a series of appropriate probes. From these isotherms, we planned to derive the energy distribution function and the energy of formation of a monolayer for the probe compounds. The probes should be chosen after consideration of the nature of the binding agent, the surfactant and the solvent used in the ceramic processing, as well as the known chemical properties of the solid surface, since the relationship between the surface energy of the powder and the properties of these chemicals is of great importance.

The characteristics of the adsorption energy distribution functions and the monolayer formation energies of probe compounds on a given surface should be related to the performance of samples of the corresponding material in actual production. If the distribution function differs for products which perform well and for those which behave poorly, the development of a quality control program for the high technology ceramic industry will be possible.

\section{B. Proposal objectives.}

our original objectives were:

1 - The development of a procedure for the preparation of porous layer open tubular columns for gas chromatography using ceramic powders or other fine particles (Sections IIB and IIC-1). 
2 - The development of an experimental protocol and of the computational tools needed to derive an adsorption isotherm from the chromatogram of a large size probe-sample (Section IID).

3 - The development of the theoretical tools required for the determination of an adsorption energy distribution function from an isotherm (Section IIE-1).

4 - The development of the computation programs needed to derive the adsorption energy distribution and the energy of formation of a monolayer from an experimental adsorption isotherm (section IIE-2).

5 - The validation of the entire methodology (Sections IIC-2, IID-2 and IIF).

6 - The determination of surface energy distribution functions for samples of various origins (Section IIF).

These objectives are compared to our results in section IIG.

\section{Evaluation of Technical Requirements.}

As explained in the original research proposal, the development of methods to manufacture porous-layer open-tubular (PLOT) columns whose wall is coated with a uniform, stable and sufficiently thick coating of the ceramic particles studied was one of the main requirements of the project. This condition has been met and the data derived from the band profiles are highly reproducible.

Most commercial gas chromatographs are designed to operate with open tubular columns and will work satisfactorily with our columns. However, we have particular requirements regarding the control and measurement of the experimental parameters, and we need rapid data acquisition and handling. Some minor modifications of the equipment were necessary. Also we had to develop programmable injection sequences, chromatographic techniques which do not demand equilibration or quantitative vapor mixing, storage of data in reasonably sized files, and procedures for data reduction involving minimum user intervention. These project requirements have also been met as we show below.

Finally, the calculation procedures must be robust, i.e., the adsorption energy distribution should be independent of minor fluctuations of the experimental data, especially of the drift and noise of the detector signal. This entire procedure must be properly validated. 


\section{II - REPORT OF TECHNICAI PROGRESS}

During the first project year (1988) we developed the basic experimental methods needed for the surface property measurements on which this study is based. We modified our gas chromatograph and developed new procedures for manufacturing porous layer open tubular columns. We developed the theory for fast isotherm determinations from chromatographic data and tested new computational methods.

During the second project year (1989) we developed the most important theoretical and experimental tools required for the successful completion of the project. We improved the column manufacturing method, completed the development of the various procedures involved in the determination of equilibrium isotherms from the chromatographic band profiles obtained with large probe samples and developed a new, more rigorous approach relating the equilibrium isotherm to the surface energy distribution.

During the third project year (1990) we completed the development of the required tools and streamlined the entire procedure of data acquisition and handling. We have (i) achieved the theoretical development of a procedure for the derivation of the energy distribution function from the experimental isotherm; (ii) written the corresponding programs and found the algorithms needed to ensure the convergence and stability of the calculations; (iii) streamlined the entire procedure of data handling which starts from the chromatogram recorded and leads to the energy distribution; (iv) verified the robustness of the whole procedure; and (v) validated the procedure.

During the last project year, we systematically acquired data on several series of alumina samples, using two probe compounds, Diethylether and 1-Chlorobutane. We have shown that, while the adsorption energy distribution functions of Diethylether on all the samples are identical within the limits of error, the opposite is true for 1-Chlorobutane. A systematic correlation has been shown between the process performance of a ceramic sample supplied by coors (Golden, CO) and the relative importance of the highest energy mode of the trimodal energy distribution obtained with this probe. We also (i) adjusted the method of preparation of PLOT columns to the use of silica particles; (ii) began the collection of experimental data on a sample of chromatographic silica; and made a systematic comparison of the various methods found in the literature to relate gas-solid equilibrium isotherms and adsorption energy distributions. 


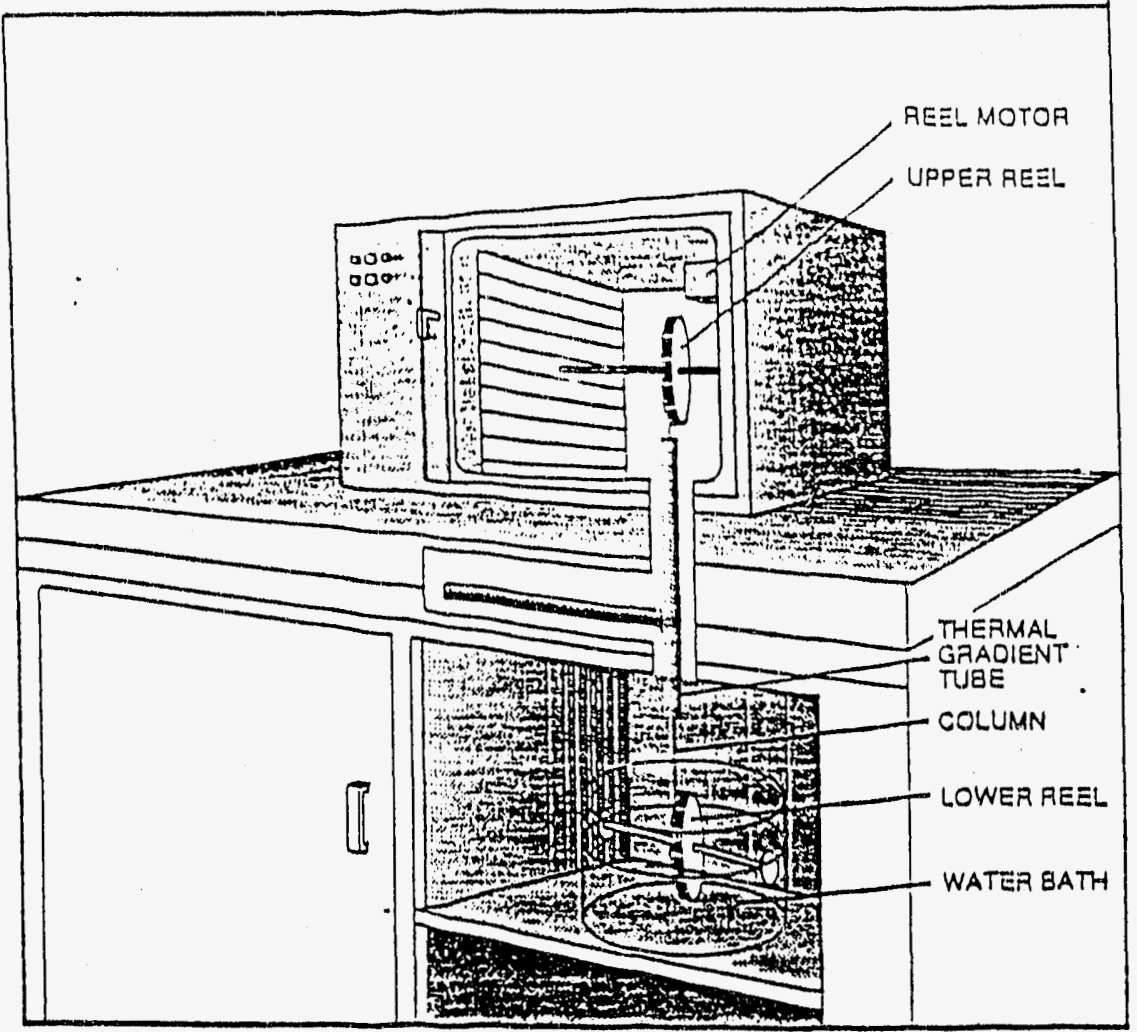

Flgure 1. Schomatics of the Apparatus used to manufacture Porous Layar Open rubular Colurms.

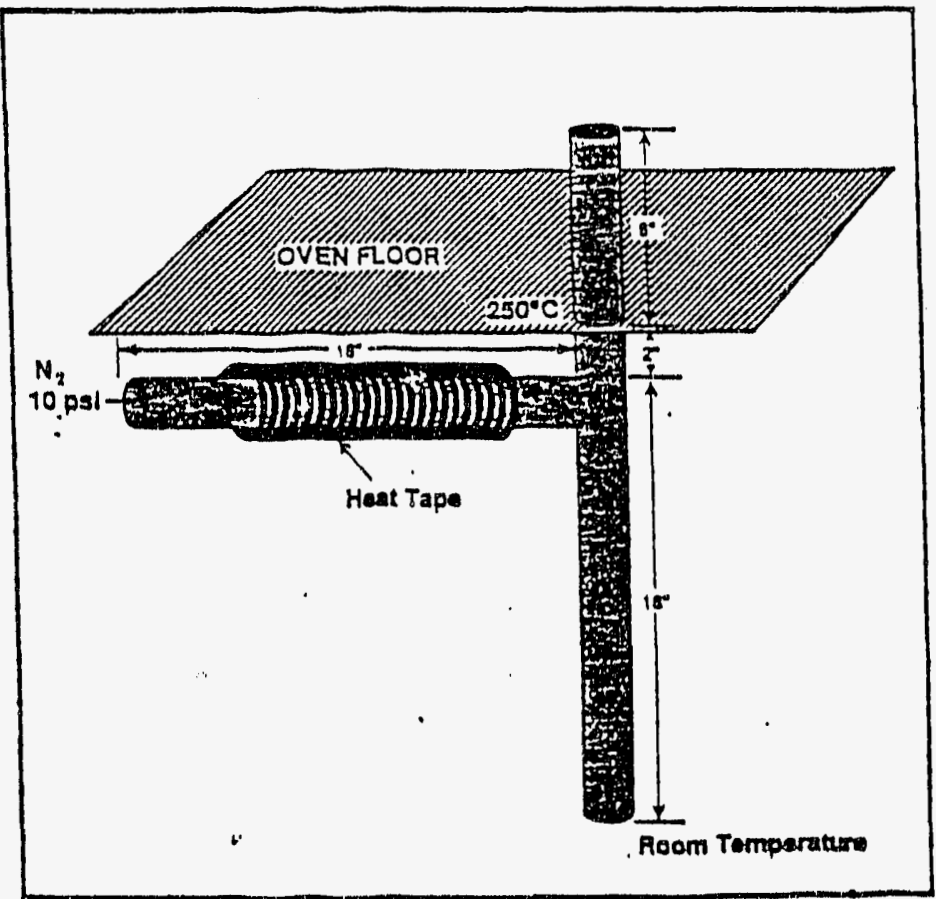

F1gure 2. Detallad achematics of the thermal gradient tube, 


\section{A. Organization of the Research.}

This research work was carried out at the Oak Ridge National Laboratory, where the Principal Investigator has a well equipped laboratory. A Perkin Elmer gas chromatograph was purchased and delivered in June 1988. The instrument to coat the inner wall of a quartz capillary with a uniform layer of particles was built there. An IBM dual-oven gas chromatograph was used for column conditioning.

All the computer calculations are carried out at the University of Tennessee Computer Center whose support and facilities have been critically helpful for the success of this work. The most cPU time demanding computer applications have been ported to the vector supercomputer on which they are now operational.

Dr. Eric Dose, a scientist with 12 years experience, and Mr. Jeffry Roles, a graduate student, have worked almost continuously on the project. Dr. Roles graduated in May and left the project in June. Ms. Jennifer Schudel, also a graduate student, has worked part-time on the project and left in July 1990 with a MS degree. Dr. Sadroddin Golshan-Shirazi, a post-doctoral fellow with a PhD from Georgetown University, is working on the comparison between the different procedures suggested to relate equilibrium isotherms and adsorption energy distributions. Prof. Minggao Xie, from the University of Wu-Han (PRC), is now working on the extension of the method to silica particles.

\section{B. Instrument Modifications.}

After a few minor problems were corrected, the position of the pressure sensor which helps in controlling the inlet column pressure, hence the flow rate, was moved to just upstream of the injector. The instrument has been working flawlessly ever since. The experimental conditions are extremely stable and the results have been highly reproducible, which has contributed markedly to the success of our work.

\section{Column Preparation.}

\section{Description of the Preparation Procedure.}

Porous Layer open Tubular (PLOT) columns with an inner wall coated rith a homogeneous thin layer of very small particles are made by evenly filling a $15 \mathrm{~m}$ long section of $0.53 \mathrm{~mm}$ ID fused silica with a stable slurry of these particles at low concentration (ca $1 \% \mathrm{w} / \mathrm{w}$ in a dibromoethane/triethylene glycol mixture) and removing the solvent without the settling of the particles. The filled column, closed at one end, is drawn vertically, at a constant rate, into a heated convection oven, through a long metal tube providing a smooth, linear thermal gradient (Figures 1 and 2 , on the opposite page). The 

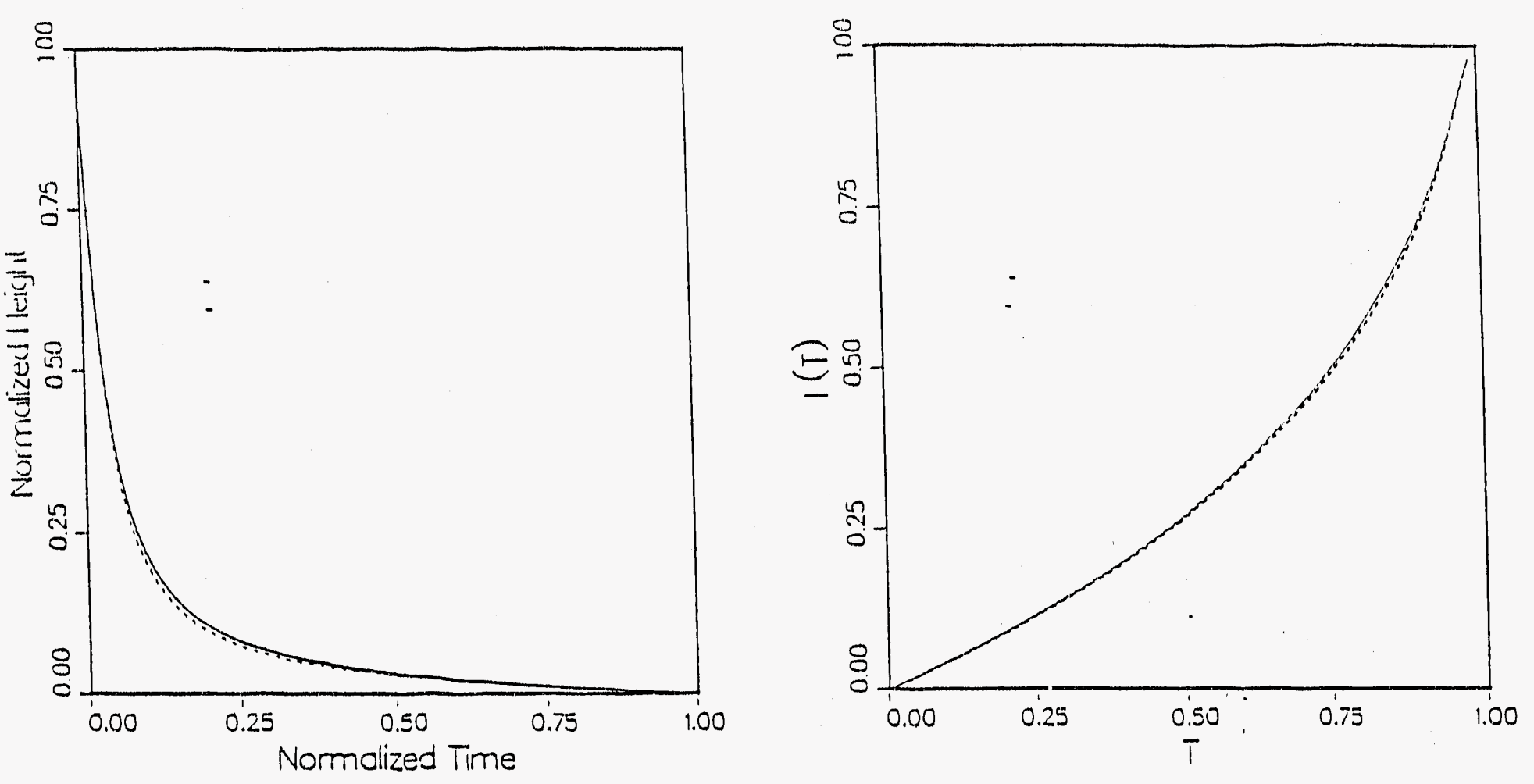

F1gure 3a. Defintition and use of tho distance botween two profiles. Left, the normalized profiles, P( $r$ ), derived from two chromatograms recorded tha same day, on the same colum, for tha same amount of Diethylether.

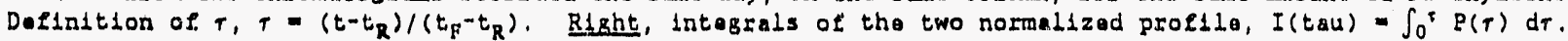
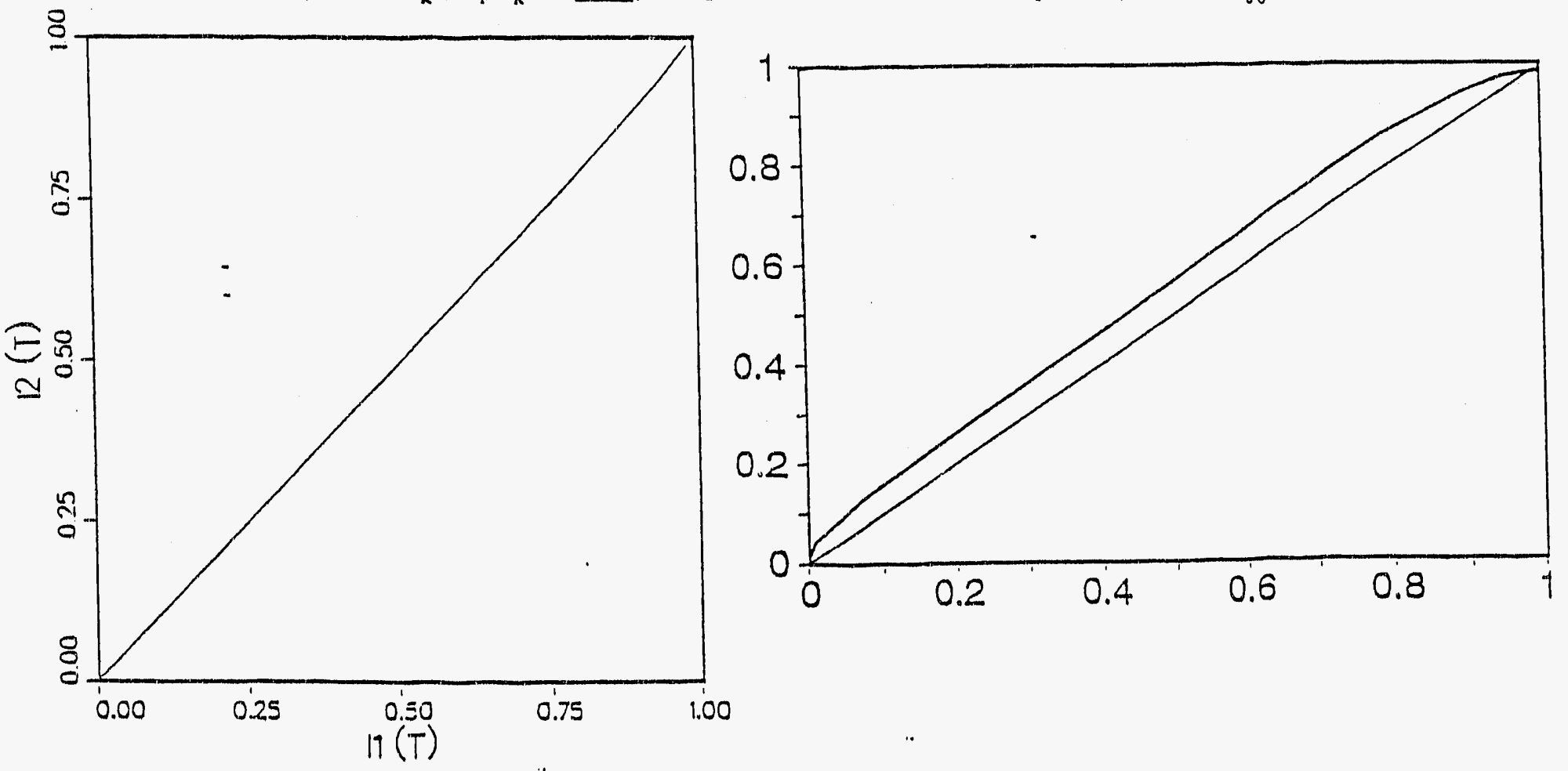

Elgure 3b. Distance botwean two proflies, Left, plot of the intagral, $I_{1}(\tau)$, of one profilo ta Figure 3 a versus the Integral. I $(\tau)$, of the other ore. Bight, Comparison betwen a Gausian and a Polsson proftila.

The distance between two profiles $1 \mathrm{~s}$ the area botween the curve and the first bisoctor. 
column is coiled on a support frame that will be used inside the GC oven, thus eliminating unnecessary handling. The temperature of the slurry-filled column yet to be drawn into the oven must be kept constant, since any expansion or contraction of the solvent will change the effective rate of feed of the solvent-vapor meniscus into the oven, and will result in a variation of the particle layer thickness.

Conditioning the PLOT column first in the convection oven and then at $350^{\circ} \mathrm{C}$ in a GC oven removes residual solvent and additives. This treatment improves the mechanical stability of the coating and removes the additives or impurities which may have been introduced during the filling and drying steps. All the details of the fabrication procedure have been described [7].

\section{Reproducibility of the Experimental Results.}

Considerable attention was paid to the development of an accurate method of determination of the adsorption isotherms of the probe compounds. The sources of errors in the acquisition of overloaded elution profiles in gas chromatography have been carefully studied [8]. The experimentation procedure has been designed to reduce as much as possible the magnitude of the errors. Quality control of the experimental results begins with the application of a new procedure which gives the distance between two different experimental profiles and permits the calculation of this distance [8] (see Figure 3, on the opposite page). The normalized profiles are defined by arbitrarily setting the peak height equal to 1 , the retention time of the band maximum (for all practical purposes, this time coincides with the retention time of the band front, see Figures 4 and 6) equal to 0 and that of the last data point different from base line equal to 1 (Figure $3 a$, left). The profile is integrated from the origin (Figure $3 a$, right). Plotting the integral $I(T)$ for one profile in Figure $3 a$ versus the other one gives the first bisector (Figure 3b, left), if the two profiles differ only by a translation and/or a similitude (e.g., two Gaussian curves). When the two profiles are different, a curve such as in Figure $3 \mathrm{~b}$, right (corresponding to the comparison between a Gaussian and a Poisson curve) is obtained.

The distance between the profiles in a series of successive experiments carried out on the same column should not exceed a threshold determined by the reproducibility of the gas chromatograph. The distance between chromatograms obtained with the same probe compounds, under the same experimental conditions, with different columns is larger than this threshold and determines the precision of the determination of the isotherm. For Diethylether on alumina, the adsorption isotherm is well accounted for by an equation summing two Langmuir terms [8]. The relative standard deviation on the coefficients of this equation is slightly less than $0.5 \%$. The process used to manufacture the PLOT columns gives columns which have very 
reproducible properties.

\section{Experimental Design and Data Estimation.}

We have described our instrument and experimental procedure in detail [7]. The column is connected to a Perkin Elmer 8500 gas chromatograph and operated isothermally, usually at $40^{\circ} \mathrm{C}$ with 1 Chlorobutane and $60^{\circ} \mathrm{C}$ with Diethylether. Helium is used as carrier gas. A large size sample is injected and the adsorption isotherm is derlved from the rear part of the band profile. In all cases so far, the isotherms are strongly convex upward, so the overloaded elution profiles have a steep front, or shock layer, and a diffuse rear. The critical steps of the procedure are the transformation of the chromatogram (time profile of the detector response) into a profile of the partial pressure of the probe at column exit as a function of the gas volume passed, the quantitation of the probe recovery, and the determination of the isotherm.

\section{Detector Calibration.}

It is important to determine the exact detector response and to verify that it is linear in the range of concentrations used. Most calibration methods, however, relate the amount injected to the peak area. We need to relate the detector signal at a certain time to the actual concentration of the probe compound in the carrier gas. We developed several procedures for this direct determination, depending whether the detector is linear or not [3].

Using the classical calibration procedure, we proved that the detector is linear under our experimental conditions. The calibration curve for the FID is constrained to proportionality of the instantaneous response and the probe concentration in the detector. only three or four chromatographic peaks are required for calibration. If necessary, other calibration curve shapes could be substituted and their parameters determined by the same method [3].

\section{Quantitation of the Desorption Recovery.}

Early experiments with alumina suggested that quasi-irreversible adsorption could occur when a probe compound is injected onto a column. An unknown but minor portion of the probe is eluted very slowly. When a temperature programmed run is performed at the end of each experiment, and started after the signal has returned to baseline for about ten minutes, a peak containing a part of the injected probe appears. Detailed analysis of the phenomenon showed, however, that the size of this peak decreases rapidly with increasing time, if the start of the temperature programmed run is delayed [8]. There is no peak if we wait for over 45 minutes before starting the temperature programmed run.

This result is in agreement with the adsorption isotherm 